\title{
EXPANSIONS OF REAL NUMBERS IN NON-INTEGER BASES
}

\author{
Danita Chunarom and Vichian LaOhakosol
}

\begin{abstract}
The works of Erdös et al. about expansions of 1 with respect to a non-integer base $q$, referred to as $q$-expansions, are investigated to determine how far they continue to hold when the number 1 is replaced by a positive number $x$. It is found that most results about $q$-expansions for real numbers greater than or equal to 1 are in somewhat opposite direction to those for real numbers less than or equal to 1 . The situation when a real number has a unique $q$-expansion, and when it has exactly two $q$-expansions are studied. The smallest base number $q$ yielding a unique $q$-expansion is determined and a particular sequence is shown, in certain sense, to be the smallest sequence whose corresponding base number $q$ yields exactly two $q$-expansions.
\end{abstract}

\section{Introduction}

Let $q \in(1,2]$. By a $q$-expansion of 1 , we mean a sequence $\left(e_{i}\right)_{i \geq 1}$ of integers in $\{0,1\}$ satisfying the equality $1=\sum_{i=1}^{\infty} e_{i} / q^{i}$. Such an expansion is not unique in general. There exist two particular expansions, constructed via the so-called greedy and lazy algorithms. In the greedy algorithm, we choose the biggest possible value for $e_{i}$, while in the lazy algorithm, we choose the smallest possible value for $e_{i}$.

In 1990, Erdös, Joo, and Komornik [4] began the work about characterizing the unique $q$-expansion of 1 for non-integer base q. In 1991, Erdös, Horváth, and Joo [3] showed that for almost all $q \in(1,2]$, there are uncountably many different $q$-expansions, and surprisingly, there exist as well uncountably many exceptional $q \in(0,1)$ for which there is only one $q$-expansion. In 1998, Komornik and Loreti [5] determined the smallest base $q \in(1,2]$ for which the $q$-expansion of 1 is unique. In 1999, Komornik and Loreti [6] gave a sufficient condition for which the number 1 has exactly two different $q$-expansions as well as using this information to construct the smallest base $q$ for which the number 1 has exactly two different $q$-expansions. In 2002, Dajani and Kraaikamp [2] studied the ergodic properties of non-greedy series expansions to non-integer

Received November 4, 2008; Revised February 6, 2009.

2000 Mathematics Subject Classification. 11A67, 11B83.

Key words and phrases. expansions of numbers, non-integer bases.

(C)2010 The Korean Mathematical Society 
bases $\beta>1$. It was shown that the so-called lazy expansion is isomorphic to the greedy expansion. Furthermore, a class of expansions to bases $\beta>1, \beta \notin \mathbb{Z}$, in between the lazy and the greedy expansions are introduced and studied. These expansions are of the form $T x=\beta x+\alpha \bmod 1$. A more recent article with contents related to this work is [7].

In this paper, our overall objective is to investigate how far the results mentioned above, excluding the cardinality and the ergodicity ones, continue to hold for the positive number $x$ replacing the number 1 . In the next section, general results about greedy and lazy $q$-expansions are derived. It is found that most results about $q$-expansions for real numbers greater than or equal to 1 are in somewhat opposite direction to those for real numbers less than or equal to 1 , which illustrate the remarkable standing of the number 1 in this regard. Through the concept of U-sequences, we then investigate the situation when a real number has unique $q$-expansion and determine the smallest such base. Finally, the situation with exactly two $q$-expansions is studied and a particular sequence, first treated in [6], which becomes in certain sense the smallest sequence for certain positive number with corresponding base $q$ yielding exactly two $q$-expansions is considered.

Let $q \in(1,2]$. By an expansion with respect to $q$, or $q$-expansion, of a positive real number $x$ we mean a sequence $\left(e_{i}\right)_{i \geq 1} \subseteq\{0,1\}$ satisfying

$$
\sum_{i=1}^{\infty} \frac{e_{i}}{q^{i}}=x .
$$

It is easily checked that $x$ has an expansion if and only if $0 \leq x \leq 1 /(q-1)$.

The lexicographical order $\prec$ is defined as follows: given two real sequences $\left(a_{i}\right)$ and $\left(b_{i}\right)$, we write $\left(a_{i}\right) \prec\left(b_{i}\right)$ or $a_{1} a_{2} \cdots \prec b_{1} b_{2} \cdots$ if there exists a positive integer $n$ such that $a_{i}=b_{i}$ for all $i<n$, but $a_{n}<b_{n}$. It is easily checked that this is a complete ordering.

Using this lexicographical order, we now define three special sequences, termed D-, U- and T-sequences. The notions of these three sequences were first considered by Komornik and Loreti [6].

A sequence $\left(a_{i}\right)_{i \geq 1} \subseteq\{0,1\}$ is called a D-sequence if

$$
\left(a_{n+i}\right) \prec\left(a_{i}\right) \text { whenever } a_{n}=0 \text {. }
$$

A sequence $\left(a_{i}\right)_{i \geq 1} \subseteq\{0,1\}$ is called a U-sequence if

$$
\left(a_{n+i}\right) \prec\left(a_{i}\right) \text { whenever } a_{n}=0 \text { (i.e., being a D-sequence) }
$$

and

$$
\left(\overline{a_{n+i}}\right):=\left(1-a_{n+i}\right) \prec\left(a_{i}\right) \text { whenever } a_{n}=1,
$$

where for brevity we write $\overline{\varepsilon_{i}}$ for $1-\varepsilon_{i}$ and $\bar{s}$ for $\overline{\varepsilon_{1} \varepsilon_{2} \cdots}$ if $s=\left(\varepsilon_{i}\right) \subseteq\{0,1\}$. If $\left(a_{i}\right)$ begins with $N(\geq 2)$ consecutive 1 digits and if there are neither $N$ consecutive 1 digits, nor $N$ consecutive 0 digits later, then it is easily checked that $\left(a_{i}\right)$ is a $\mathrm{U}$-sequence. 
A sequence $\left(e_{i}\right)_{i \geq 1} \subseteq\{0,1\}$ is called a T-sequence if the following three conditions hold:

(1) $\left(e_{n+i}\right) \prec\left(e_{i}\right)$ whenever $e_{n}=0$ (i.e., $\left(e_{i}\right)$ is D-sequence);

(2) there exists a positive integer $m$ such that $e_{m}=1$, and

(3) there exists a sequence $\left(\varepsilon_{i}\right)_{i \geq 1} \subseteq\{0,1\}$ defined by $e_{i+m}+\varepsilon_{i} \in\{0,1\}(i \geq$ $1)$, such that if the sequence $\left(\delta_{i}\right)_{i \geq 1} \subseteq\{0,1\}$ is defined by

$$
\delta_{i}= \begin{cases}e_{i} & \text { if } i<m \\ 0 & \text { if } i=m \\ e_{i}+\varepsilon_{i-m} & \text { if } i>m,\end{cases}
$$

then the following three requirements hold:

$$
\begin{aligned}
& \overline{\delta_{n+1} \delta_{n+2} \cdots} \prec e_{1} e_{2} \cdots \text { whenever } \delta_{n}=1, \\
& \overline{e_{n+1} e_{n+2} \cdots} \prec e_{1} e_{2} \cdots \text { whenever } e_{n}=1 \text { and } n>m, \\
& \delta_{n+1} \delta_{n+2} \cdots \prec e_{1} e_{2} \cdots \text { whenever } \delta_{n}=0 \text { and } n>m .
\end{aligned}
$$

Komornik and Loreti [6] showed that if $\left(e_{i}\right)$ is a T-sequence with $e_{i}=\varepsilon_{i}$, then there exists a $q \in(1,2]$ such that 1 has exactly two expansions.

A real number $q \in(1,2]$ is called a T-base number if there exists a positive real number $x$ with exactly two different $q$-expansions.

As a general, preliminary result, we have:

Theorem 1.1. Let $\left(e_{i}\right) \subseteq\{0,1\}$. Then the map

$$
q \mapsto \sum_{i \geq 1} e_{i} / q^{i}
$$

is continuous and strictly decreasing from the interval $(1,2]$ onto the interval $\left[\sum_{i \geq 1} e_{i} / 2^{i}, \sum_{i \geq 1} e_{i}\right)$.

Proof. Let $q \in(1,2]$ and $F(q)=\sum_{i \geq 1} e_{i} / q^{i}$. That this map is strictly decreasing is clear. If $q_{1}<q_{2}$, then

$$
\left|F\left(q_{1}\right)-F\left(q_{2}\right)\right|=\sum_{i \geq 1}\left|\frac{e_{i}\left(q_{1}^{i}-q_{2}^{i}\right)}{q_{1}^{i} q_{2}^{i}}\right| \leq \frac{\left|q_{1}-q_{2}\right|}{q_{1} q_{2}} \sum_{i \geq 1} \frac{i}{q_{1}^{i-1}},
$$

showing that $F$ is continuous. That this map is onto follows from the intermediate value theorem for continuous functions.

\section{Greedy expansions}

Let $q \in(1,2]$ and $x \in[0,1 /(q-1)]$. We define the greedy $q$-expansion $\left(a_{i}\right) \subseteq\{0,1\}$ of $x$ as follows: if for some positive integer $n$, the numbers $a_{i}$ are defined for all $i<n$, then set $a_{n}=1$ whenever

$$
\sum_{i=1}^{n-1} \frac{a_{i}}{q^{i}}+\frac{1}{q^{n}} \leq x
$$


and $a_{n}=0$ otherwise, where the summation is taken as 0 if $n=1$.

Our next result reveals some intrinsic relations between the greedy $q$-expansion of a number in $[1,1 /(q-1)]$ and that of any non-negative real number in $[0,1 /(q-1)]$.

Theorem 2.1. Let $q \in(1,2], y \geq 1$ and let $\left(e_{i}\right)$ be the greedy $q$-expansion of $y$.

(a) The greedy $q$-expansion, $\left(a_{i}\right)$, of any $x \in[0,1 /(q-1)]$ satisfies

$$
a_{n+1} a_{n+2} \cdots \prec e_{1} e_{2} \cdots \text { whenever } a_{n}=0 \text {. }
$$

(b) If the sequence $\left(e_{i}\right)$ is finite with a last nonzero digit $e_{k}$, then no greedy $q$-expansion is eventually periodic with the period $e_{1} e_{2} \cdots e_{k-1}\left(e_{k}-1\right)$.

Proof. (a) Assume that $a_{n}=0$. If $\left(a_{n+i}\right) \succ\left(e_{i}\right)$, then there exists an integer $k$ such that $a_{n+i}=e_{i}$ for $i=1,2, \ldots, k-1$, but $a_{n+k}>e_{k}$. Thus $e_{k}=0$ and $a_{n+k}=1$ and so, by the definition of greedy $q$-expansion of $y$,

$$
\sum_{i=1}^{k-1} \frac{e_{i}}{q^{i}}+\frac{1}{q^{k}}>y
$$

Thus

$$
\sum_{i=1}^{\infty} \frac{a_{n+i}}{q^{i}} \geq \sum_{i=1}^{k-1} \frac{a_{n+i}}{q^{i}}+\frac{1}{q^{k}}=\sum_{i=1}^{k-1} \frac{e_{i}}{q^{i}}+\frac{1}{q^{k}}>y
$$

and so

$x=\sum_{i=1}^{n-1} \frac{a_{i}}{q^{i}}+\frac{a_{n}}{q^{n}}+\frac{1}{q^{n}}\left(\frac{a_{n+1}}{q}+\frac{a_{n+2}}{q^{2}}+\cdots\right)>\sum_{i=1}^{n-1} \frac{a_{i}}{q^{i}}+\frac{a_{n}}{q^{n}}+\frac{y}{q^{n}} \geq \sum_{i=1}^{n-1} \frac{a_{i}}{q^{i}}+\frac{1}{q^{n}}$,

contradicting the definition of the greedy $q$-expansion of $x$ (because $a_{n}=0$ ).

If $\left(a_{n+i}\right)=\left(e_{i}\right)$, then $a_{n+i}=e_{i}$ for all $i \geq 1$. Thus

$$
\begin{aligned}
x & =\sum_{i=1}^{n-1} \frac{a_{i}}{q^{i}}+\frac{a_{n}}{q^{n}}+\frac{1}{q^{n}}\left(\frac{a_{n+1}}{q}+\frac{a_{n+2}}{q^{2}}+\cdots\right) \\
& =\sum_{i=1}^{n-1} \frac{a_{i}}{q^{i}}+\frac{a_{n}}{q^{n}}+\frac{1}{q^{n}}\left(\frac{e_{1}}{q}+\frac{e_{2}}{q^{2}}+\cdots\right) \\
& =\sum_{i=1}^{n-1} \frac{a_{i}}{q^{i}}+\frac{a_{n}}{q^{n}}+\frac{y}{q^{n}} \\
& \geq \sum_{i=1}^{n-1} \frac{a_{i}}{q^{i}}+\frac{1}{q^{n}},
\end{aligned}
$$

again contradicting the definition of the greedy $q$-expansion of $x$ (because $a_{n}=$ $0)$. 
(b) Assume on the contrary that the greedy $q$-expansion $\left(a_{i}\right)$ of some $x \in$ $[0,1 /(q-1)]$ is eventually periodic with period $e_{1} e_{2} \cdots e_{k-1}\left(e_{k}-1\right)$. Since

we have

$$
y-\frac{1}{q^{k}}=\frac{e_{1}}{q^{1}}+\cdots+\frac{e_{k}-1}{q^{k}},
$$

$$
\begin{aligned}
x= & \left(\frac{a_{1}}{q}+\cdots+\frac{a_{r}}{q^{r}}\right)+\frac{1}{q^{r}}\left(\frac{e_{1}}{q}+\cdots+\frac{e_{k}-1}{q^{k}}\right) \\
& +\left(\frac{e_{1}}{q}+\cdots+\frac{e_{k}-1}{q^{k}}\right)\left(\frac{1}{q^{r+k}}+\frac{1}{q^{r+2 k}}+\cdots\right) \\
= & \left(\frac{a_{1}}{q}+\cdots+\frac{a_{r}}{q^{r}}\right)+\frac{1}{q^{r}}\left(\frac{e_{1}}{q}+\cdots+\frac{e_{k}-1}{q^{k}}\right)+\left(y-\frac{1}{q^{k}}\right)\left(\frac{\frac{1}{q^{r+k}}}{1-\frac{1}{q^{k}}}\right) \\
\geq & \left(\frac{a_{1}}{q^{1}}+\cdots+\frac{a_{r}}{q^{r}}\right)+\frac{1}{q^{r}}\left(\frac{e_{1}}{q^{1}}+\cdots+\frac{e_{k}-1}{q^{k}}\right)+\left(1-\frac{1}{q^{k}}\right)\left(\frac{\frac{1}{q^{r+k}}}{1-\frac{1}{q^{k}}}\right) \\
= & \left(\frac{a_{1}}{q^{1}}+\cdots+\frac{a_{r}}{q^{r}}\right)+\frac{1}{q^{r}}\left(\frac{e_{1}}{q^{1}}+\cdots+\frac{e_{k}}{q^{k}}\right)=\sum_{i=1}^{r+k-1} \frac{a_{i}}{q^{i}}+\frac{1}{q^{r+k}},
\end{aligned}
$$

contradicting the definition of the $q$-greedy expansion of $x$ (because $a_{r+k}=$ $0)$.

Remarks. 1) The case where $y=1$ is Lemmas 2(a) in [2] and Lemma 1.4(a) in $[5]$.

2) The converse of Theorem 2.1(a) is not true, i.e., there exists an $x \in$ $[0,1 /(q-1)]$, whose $q$-expansion, $\left(a_{i}\right)$, satisfies the condition $(2.1)$, but this expansion is not the greedy $q$-expansion of $x$, as seen in the following example.

Example. Take $q=\frac{9}{5}$ and $x=y=\frac{27199387096045}{22876792454961}=\frac{27199387096045}{9^{14}} \geq 1$. Here

$$
\left(e_{i}\right)=(1,1,1,1,1,0,0,0,1,0,0,0,0,0,0,0,0,0, \ldots),
$$

the expression holding up to the first eighteen digits, is the greedy $q$-expansion of $x=y$ and

$$
\left(a_{i}\right)=(1,1,1,1,1,0,0,0,0,1,1,0,1,1)
$$

is a finite $q$-expansion of $x=y$ which satisfies the condition $(2.1)$, but $\left(a_{i}\right)$ is not a greedy $q$-expansion.

Next we derive more characterizations of greedy $q$-expansions.

Theorem 2.2. Let $q \in(1,2]$. A sequence $\left(a_{i}\right)$ is the greedy $q$-expansion of $x$ if and only if $\sum_{i=1}^{\infty} a_{k+i} / q^{i}<1$ whenever $a_{k}=0$.

Proof. Let $\left(a_{i}\right)$ be the greedy $q$-expansion of $x$ and assume $a_{k}=0$. By definition,

$$
\sum_{i=1}^{k-1} \frac{a_{i}}{q^{i}}+\frac{1}{q^{k}}>x
$$


and so

$$
\frac{1}{q^{k}}>\sum_{i=k}^{\infty} \frac{a_{i}}{q^{i}}=\sum_{i=k+1}^{\infty} \frac{a_{i}}{q^{i}}=\sum_{i=1}^{\infty} \frac{a_{k+i}}{q^{k+i}} .
$$

The required inequality follows after multiplying by $q^{k}$.

Assume $\sum_{i=1}^{\infty} a_{k+i} / q^{i}<1$ whenever $a_{k}=0$. If $a_{n}=1$, then

$$
x=\sum_{i=1}^{n-1} \frac{a_{i}}{q^{i}}+\frac{1}{q^{n}}+\sum_{i>n} \frac{a_{i}}{q^{i}} \geq \sum_{i=1}^{n-1} \frac{a_{i}}{q^{i}}+\frac{1}{q^{n}} .
$$

If $a_{n}=0$, then $\sum_{i=1}^{\infty} a_{n+i} / q^{i}<1$, and so $\sum_{i=1}^{\infty} a_{n+i} / q^{n+i}<1 / q^{n}$. Thus

$$
x=\sum_{i=1}^{\infty} \frac{a_{i}}{q^{i}}=\sum_{i \neq n} \frac{a_{i}}{q^{i}}=\sum_{i=1}^{n-1} \frac{a_{i}}{q^{i}}+\sum_{i=1}^{\infty} \frac{a_{n+i}}{q^{n+i}}<\sum_{i=1}^{n-1} \frac{a_{i}}{q^{i}}+\frac{1}{q^{n}},
$$

i.e., $\left(a_{i}\right)$ is the greedy $q$-expansion of $x$.

Remark. Theorem 2.2 is Lemma 1(a) in [2], but the proof here is different.

Theorem 2.3. Let $q \in(1,2]$.

(a) Let $\left(e_{i}\right)$ be an infinite q-expansion of $y \in[0,1]$ and let $\left(a_{i}\right)$ be a $q$ expansion of $x \in[0,1 /(q-1)]$. If the condition $(2.1)$ holds, then $\left(a_{i}\right)$ is the greedy q-expansion of $x$.

(b) Let $\left(e_{i}\right)$ be a q-expansion of $y \in[0,1]$ and let $\left(a_{i}\right)$ be a finite q-expansion of $x \in[0,1 /(q-1)]$. If the condition $(2.1)$ holds, then $\left(a_{i}\right)$ is the greedy $q$ expansion of $x$.

(c) Let $\left(e_{i}\right)$ be a finite q-expansion of $y \in[0,1]$ and denote by $e_{k}$ its last nonzero element. Let $\left(a_{i}\right)$ be a q-expansion of $x \in[0,1 /(q-1)]$. Assume $(2.1)$ holds.

(c.1) If $y<1$, then $\left(a_{i}\right)$ is the greedy $q$-expansion of $x$.

(c.2) If $y=1$ and assume that $\left(a_{i}\right)$ is not eventually periodic with period $e_{1} \cdots e_{k-1}\left(e_{k}-1\right)$, then $\left(a_{i}\right)$ is the greedy $q$-expansion of $x$.

Proof. There is nothing to prove if $a_{n}=1$, while for those $n$ with $a_{n}=0$, the results follow from Theorem 2.2 if we can show that

$$
\sum_{i=1}^{\infty} \frac{a_{n+i}}{q^{i}}<1
$$

From (2.1), there is a sequence of integers $n=k_{0}<k_{1}<\cdots$ satisfying the conditions: with $j \in \mathbb{N}$,

$$
a_{k_{j-1}+i}=e_{i} \text { for all } 1 \leq i<k_{j}-k_{j-1} \text { and } a_{k_{j}}<e_{k_{j}-k_{j-1}} .
$$

(a) If the sequence $\left(e_{i}\right)$ is infinite, then

$$
\frac{1}{q^{n}} \sum_{i=1}^{\infty} \frac{a_{n+i}}{q^{i}}=\sum_{j=1}^{\infty}\left(\sum_{i=1}^{k_{j}-k_{j-1}} \frac{a_{k_{j-1}+i}}{q^{k_{j-1}+i}}\right)=\sum_{j=1}^{\infty}\left(\sum_{i=1}^{k_{j}-k_{j-1}} \frac{e_{i}}{q^{k_{j-1}+i}}-\frac{1}{q^{k_{j}}}\right)
$$




$$
<\sum_{j=1}^{\infty}\left(\frac{y}{q^{k_{j-1}}}-\frac{1}{q^{k_{j}}}\right) \leq \frac{1}{q^{k_{0}}}
$$

proving (2.2).

(b) If the sequence $\left(a_{i}\right)$ is finite, assume that there exits a positive integer $m$ satisfying $a_{i}=0$ for all $i>k_{m}$. Now

$$
\begin{aligned}
\frac{1}{q^{n}} \sum_{i=1}^{\infty} \frac{a_{n+i}}{q^{i}} & =\sum_{j=1}^{m}\left(\sum_{i=1}^{k_{j}-k_{j-1}} \frac{a_{k_{j-1}+i}}{q^{k_{j-1}+i}}\right)=\sum_{j=1}^{m}\left(\sum_{i=1}^{k_{j}-k_{j-1}} \frac{e_{i}}{q^{k_{j-1}+i}}-\frac{1}{q^{k_{j}}}\right) \\
& \leq \sum_{j=1}^{m}\left(\frac{y}{q^{k_{j-1}}}-\frac{1}{q^{k_{j}}}\right) \leq \frac{1}{q^{k_{0}}}-\frac{1}{q^{m}}<\frac{1}{q^{k_{0}}},
\end{aligned}
$$

proving (2.2).

(c) If the sequence $\left(e_{i}\right)$ is finite, proceeding as in the proof of (a) leads to (2.3) with strict inequality being now non-strict. Observe that $e_{k_{j}-k_{j-1}}=1$ so $k_{j}-k_{j-1} \leq k$. A closer inspection of the proof reveals that we obtain equality exactly when $y=1$ and $k_{j}-k_{j-1}=k$ for every $j$, i.e., when the sequence $\left(a_{n+i}\right)$ is periodic with period $e_{1} \cdots e_{k-1}\left(e_{k}-1\right)$. This contradicts the fact that $\left(a_{i}\right)$ is not eventually periodic with period $e_{1} \cdots e_{k-1}\left(e_{k}-1\right)$. Hence, $\left(a_{i}\right)$ is the greedy $q$-expansion of $x$.

Remarks. 1) Theorem 2.3 (a), (b) is Lemma 3 in [2] and the proofs given here are the same. Lemma 1.5 (a) in [5] is a special case of Theorem 2.3 (c.2) above.

2) The converse of Theorem 2.3 (c.1) is not true, i.e., there exist $y$ with finite $q$-expansion $\left(e_{i}\right)$, and $x$ with greedy $q$-expansion $\left(a_{i}\right)$, such that $\left(a_{i}\right)$ does not satisfy the condition (2.1) as seen in the following example.

Example. Take $q=4 / 3$. We have

$$
\left(a_{i}\right)=(1,0,0,0,0,0,1,0,0,0,0,0,0,0,1,0,0,0,0,0,1,0,0,0,1,0,0,0,0,0,0,0,0,0,0,0,0,1)
$$

is the greedy expansion of $x=\frac{68001788610072039914841}{75557863725914323419136}$. Taking $y=x<1$, we get $\left(a_{i}\right)=\left(e_{i}\right)$. Note that $\left(a_{i}\right)$ does not satisfy the condition $(2.1)$.

Theorem 2.4. Let $q, q^{\prime} \in(1,2], x \in[0,1 /(q-1)] \cap\left[0,1 /\left(q^{\prime}-1\right)\right]$. Let $\left(e_{i}\right)$ and $\left(e_{i}^{\prime}\right)$ be the greedy $q$-expansion, respectively, greedy $q^{\prime}$-expansion of $x$. If $q<q^{\prime}$, then $\left(e_{i}\right) \prec\left(e_{i}^{\prime}\right)$.

Proof. Suppose that the conclusion is false. We have two possible cases.

Case 1: $\left(e_{i}\right)=\left(e_{i}^{\prime}\right)$. Thus $x=\sum_{i=1}^{\infty} e_{i}^{\prime} /\left(q^{\prime}\right)^{i}<\sum_{i=1}^{\infty} e_{i} / q^{i}=x$, a contradiction. Case 2: $\left(e_{i}\right) \succ\left(e_{i}^{\prime}\right)$. Thus there exists an integer $n$ such that $e_{i}=e_{i}^{\prime}$ for all $1 \leq i<n$ but $e_{n}>e_{n}^{\prime}$. We must have $e_{n}=1$ and $e_{n}^{\prime}=0$. By the definition of the greedy $q$-expansion,

$$
\sum_{i=1}^{n-1} \frac{e_{i}^{\prime}}{q^{\prime i}}+\frac{1}{q^{\prime n}} \leq \sum_{i=1}^{n-1} \frac{e_{i}}{q^{i}}+\frac{1}{q^{\prime n}}<\sum_{i=1}^{n-1} \frac{e_{i}}{q^{i}}+\frac{1}{q^{n}} \leq x,
$$

contradicting the definition of greedy $q^{\prime}$-expansion of $x$ as $e_{n}^{\prime}=0$. 


\section{Lazy expansions}

Let $q \in(1,2], y \in[0,1 /(q-1)]$. The lazy q-expansion $\left(b_{i}\right)$ of $y$ is defined as follows: if for some positive integer $n$ the numbers $b_{i}$ are defined for all $i<n$, then set $b_{n}=0$ whenever

$$
\sum_{i=1}^{n-1} \frac{b_{i}}{q^{i}}+\sum_{i>n} \frac{1}{q^{i}} \geq y
$$

and set $b_{n}=1$ otherwise, where the summation is taken as 0 if $n=1$.

Lazy $q$-expansions enjoy two simple properties which we now describe.

Property L1. A real number $y \in[0,1 /(q-1)]$ has $\left(b_{i}\right)$ as its lazy $q$-expansion if and only if the sequence $\left(a_{i}\right):=\left(1-b_{i}\right)$ is the greedy $q$-expansion of $\frac{1}{q-1}-y$ (This "duality" property implies that every $y \in[0,1 /(q-1)]$ has a lazy $q$ expansion).

Proof. First observe that

$\left(b_{i}\right)$ is a $q$-expansion of $y \Leftrightarrow \sum_{i=1}^{\infty} b_{i} / q^{i}=y \Leftrightarrow \sum_{i=1}^{\infty}\left(1-b_{i}\right) / q^{i}=\frac{1}{q-1}-y \Leftrightarrow$ $\left(1-b_{i}\right)$ is a $q$-expansion of $\frac{1}{q-1}-y$.

Assume that $\left(b_{i}\right)$ is the lazy $q$-expansion of $y$. If $1-b_{n}=0$, then

$$
y>\sum_{i=1}^{n-1} \frac{b_{i}}{q^{i}}+\sum_{i>n} \frac{1}{q^{i}}
$$

and so

$$
\frac{1}{q-1}-y<\frac{1}{q-1}-\sum_{i=1}^{n-1} \frac{b_{i}}{q^{i}}-\sum_{i>n} \frac{1}{q^{i}}=\sum_{i=1}^{n-1} \frac{1-b_{i}}{q^{i}}+\frac{1}{q^{n}} .
$$

If $1-b_{n}=1$, then $y \leq \sum_{i=1}^{n-1} b_{i} / q^{i}+\sum_{i>n} 1 / q^{i}$, and so

$$
\frac{1}{q-1}-y \geq \frac{1}{q-1}-\sum_{i=1}^{n-1} \frac{b_{i}}{q^{i}}-\sum_{i>n} \frac{1}{q^{i}}=\sum_{i=1}^{n-1} \frac{1-b_{i}}{q^{i}}+\frac{1}{q^{n}} .
$$

Thus $\left(1-b_{i}\right)$ is the greedy $q$-expansion of $\frac{1}{q-1}-y$.

Assume that $\left(1-b_{i}\right)$ is the greedy $q$-expansion of $\frac{1}{q-1}-y$. If $b_{n}=0$, then $\frac{1}{q-1}-y \geq \sum_{i=1}^{n-1}\left(1-b_{i}\right) / q^{i}+1 / q^{n}$, and so

$$
y \leq \frac{1}{q-1}-\sum_{i=1}^{n-1} \frac{1-b_{i}}{q^{i}}-\frac{1}{q^{n}}=\sum_{i=1}^{n-1} \frac{b_{i}}{q^{i}}+\sum_{i>n} \frac{1}{q^{i}} .
$$

If $b_{n}=1$, then $\frac{1}{q-1}-y<\sum_{i=1}^{n-1}\left(1-b_{i}\right) / q^{i}+1 / q^{n}$, and so $y>\sum_{i=1}^{n-1} b_{i} / q^{i}+$ $\sum_{i>n} 1 / q^{i}$. Thus $\left(b_{i}\right)$ is the lazy $q$-expansion of $y$.

Property L2. If $\left(a_{i}\right)$ and $\left(b_{i}\right)$ are the greedy and lazy $q$-expansions, respectively, of $x$, and if there exists another $q$-expansion $\left(c_{i}\right)$ of $x$, then

$$
\left(b_{i}\right) \preceq\left(c_{i}\right) \preceq\left(a_{i}\right)
$$


(In other words, the greedy $q$-expansion is the greatest $q$-expansion and the lazy $q$-expansion is the smallest $q$-expansion of $x$ lexicographically).

Proof. Let $\left(a_{i}\right)$ and $\left(b_{i}\right)$ be the greedy, respectively, lazy $q$-expansions of $x$ and let $\left(c_{i}\right)$ be another $q$-expansion of $x$.

To show that $\left(b_{i}\right) \preceq\left(c_{i}\right)$, assume $\left(b_{i}\right) \succ\left(c_{i}\right)$. Then there exists an integer $n$ such that $b_{i}=c_{i}$ for all $1 \leq i<n$ but $b_{n}>c_{n}$. Thus $b_{n}=1$ and $c_{n}=0$. By the definition of lazy $q$-expansion, we have

$$
\sum_{i=1}^{n-1} \frac{b_{i}}{q^{i}}+\sum_{i \geq n+1} \frac{1}{q^{i}}<x=\sum_{i=1}^{\infty} \frac{c_{i}}{q^{i}}=\sum_{i=1}^{n-1} \frac{c_{i}}{q^{i}}+\sum_{i \geq n} \frac{c_{i}}{q^{i}} .
$$

Thus

$$
\sum_{i \geq n+1} \frac{1}{q^{i}}<\sum_{i \geq n} \frac{c_{i}}{q^{i}}=\sum_{i \geq n+1} \frac{c_{i}}{q^{i}}
$$

contradicting the definition of the sequence $\left(c_{i}\right) \subseteq\{0,1\}$.

To show that $\left(c_{i}\right) \preceq\left(a_{i}\right)$, assume $\left(c_{i}\right) \succ\left(a_{i}\right)$. Then there exists an integer $n$ such that $c_{i}=a_{i}$ for all $1 \leq i<n$ but $c_{n}>a_{n}$. Thus $c_{n}=1$ and $a_{n}=0$. By the definition of greedy $q$-expansion, we have

$$
\sum_{i=1}^{n-1} \frac{a_{i}}{q^{i}}+\frac{1}{q^{n}}>x=\sum_{i=1}^{\infty} \frac{c_{i}}{q^{i}}=\sum_{i=1}^{n-1} \frac{c_{i}}{q^{i}}+\sum_{i \geq n} \frac{c_{i}}{q^{i}},
$$

which implies $0>\sum_{i \geq n+1} \frac{c_{i}}{q^{i}}$, again contradicting the definition of the sequence $\left(c_{i}\right) \subseteq\{0,1\}$

Remark. Properties L1 and L2 are well known and have appeared in several articles and with quite short proofs, e.g. [6] and [1], where in the latter paper simple and short dynamical proofs are given. We give the above proofs for two reasons; first, they are elementary and second, to make this exposition self-contained.

We next derive further characterizations of lazy $q$-expansions.

Theorem 3.1. Let $q \in(1,2], x \in[0,1 /(q-1)]$. Then $\left(b_{i}\right)$ is the lazy $q$ expansion of $x$ if and only if $\sum_{i=1}^{\infty}\left(1-b_{k+i}\right) / q^{i}<1$ whenever $b_{k}=1$.

Proof. Let $\left(b_{i}\right)$ be the lazy $q$-expansion of $x$. Assuming $b_{k}=1$, we get

$$
\sum_{i=1}^{k} \frac{b_{i}}{q^{i}}+\sum_{i \geq k+1} \frac{1}{q^{i}}<x+\frac{1}{q^{k}}=\sum_{i=1}^{\infty} \frac{b_{i}}{q^{i}}+\frac{1}{q^{k}},
$$

and so

$$
\sum_{i=1}^{\infty} \frac{1-b_{k+i}}{q^{i}}<1
$$


Conversely, assume $\sum_{i=1}^{\infty}\left(1-b_{k+i}\right) / q^{i}<1$ whenever $b_{k}=1$. If $b_{n}=0$, then

$$
x=\sum_{i=1}^{n-1} \frac{b_{i}}{q^{i}}+\sum_{i \geq n+1} \frac{b_{i}}{q^{i}} \leq \sum_{i=1}^{n-1} \frac{b_{i}}{q^{i}}+\sum_{i \geq n+1} \frac{1}{q^{i}} .
$$

If $b_{n}=1$, then from the assumption we have $\sum_{i=1}^{\infty}\left(1-b_{n+i}\right) / q^{n+i}<1 / q^{n}$, and so $\sum_{i=1}^{\infty} 1 / q^{n+i}+\sum_{i=1}^{n} b_{i} / q^{i}<x+1 / q^{n}$, i.e., $\sum_{i=1}^{n-1} b_{i} / q^{i}+\sum_{i \geq n+1} 1 / q^{i}<x$, showing that the $q$-expansion is lazy.

Remark. Theorem 3.1 is Lemma 1(b) in [2], but the proof here is different.

Theorem 3.2. Let $\left(e_{i}\right)$ be an infinite $q$-expansion of $y \leq 1$. If another infinite $q$-expansion $\left(b_{i}\right)$ of $x \in[0,1 /(q-1)]$ satisfies the condition

$$
\left(1-b_{n+i}\right) \prec\left(e_{i}\right) \text { whenever } b_{n}=1 \text {, }
$$

then $\left(b_{i}\right)$ is the lazy q-expansion of $x$.

Proof. By Theorem 3.1, it suffices to show that if $b_{k}=1$, then

$$
\sum_{i=1}^{\infty} \frac{1-b_{k+i}}{q^{i}}<1
$$

Let $b_{k}=1$. By hypothesis, there is a sequence of integers $k=k_{0}<k_{1}<\cdots$ satisfying for each $j=1,2, \ldots$ the conditions

$$
1-b_{k_{j-1}+i}=e_{i} \text { when } 1 \leq i<k_{j}-k_{j-1} \text {, and } 1-b_{k_{j}}<e_{k_{j}-k_{j-1}} \text {. }
$$

We have

$$
\begin{aligned}
\frac{1}{q^{k}} \sum_{i=1}^{\infty} \frac{1-b_{k+i}}{q^{i}} & =\sum_{j=1}^{\infty}\left(\sum_{i=1}^{k_{j}-k_{j-1}} \frac{1-b_{k_{j-1}+i}}{q^{k_{j-1}+i}}\right)=\sum_{j=1}^{\infty}\left(\sum_{i=1}^{k_{j}-k_{j-1}} \frac{e_{i}}{q^{k_{j-1}+i}}-\frac{1}{q^{k_{j}}}\right) \\
& =\sum_{j=1}^{\infty}\left(\frac{1}{q^{k_{j-1}}} \sum_{i=1}^{k_{j}-k_{j-1}} \frac{e_{i}}{q^{i}}-\frac{1}{q^{k_{j}}}\right)<\sum_{j=1}^{\infty}\left(\frac{y}{q^{k_{j-1}}}-\frac{1}{q^{k_{j}}}\right) \leq \frac{1}{q^{k_{0}}},
\end{aligned}
$$

and the desired result follows at once.

Remark. Proposition 2.1 in [5] is a special case of Theorem 3.2 when $y=1$.

Theorem 3.3. Let $q \in(1,2],\left(e_{i}\right)$ be a finite $q$-expansion of $y \leq 1$ and denote by $e_{L}$ its last nonzero digit. If an infinite $q$-expansion $\left(b_{i}\right)$ of $x \in[0,1 /(q-1)]$ satisfies the condition (3.1) and

$$
L>\min \left\{k ; \text { for each } i \in \mathbb{N} \text {, if } b_{i}=1 \text {, then } b_{i+j} \neq e_{j} \text { when } 1 \leq j<k\right.
$$

$$
\text { and } \left.b_{i+k}=e_{k}=1\right\} \text {, }
$$

then $\left(b_{i}\right)$ is the lazy q-expansion of $x$. 
Proof. Proceeding exactly as in the proof of Theorem 3.2, we end up at (3.3) but the strict inequality now becomes non-strict. If (3.3) is an equality, then $y=1$ and more importantly, $k_{j}-k_{j-1}=L$ for each $j$ but the condition (3.4) prevents this from happening.

Remarks. Theorem 3.3 is new and complements Theorem 3.2. The condition (3.1) is not needed when $x=0$. For then $x$ has only a unique $q$-expansion which must then be (0) violating (3.1).

\section{Numbers with unique $q$-expansion and smallest base}

In this section, we first find conditions for which the greedy and lazy $q$ expansions of a fixed real number coincide, i.e., conditions for which the $q$ expansion is unique.

Theorem 4.1. If the number $\sigma \geq 1$ has a unique $q$-expansion, $\left(\varepsilon_{i}\right)$, for a given $q \in(1,2]$, then this unique q-expansion is a $U$-sequence.

Proof. Let $\sigma \geq 1$ and $\left(\varepsilon_{i}\right)$ be unique, and so is a greedy $q$-expansion. We deduce from Theorem 2.1, using $x=y=\sigma$, that $\left(\varepsilon_{n+i}\right) \prec\left(\varepsilon_{i}\right)$ whenever $\varepsilon_{n}=0$. Since $\left(\varepsilon_{i}\right)$ is also the lazy $q$-expansion of $\sigma$, by Property L1, the $q$-expansion $\left(1-\varepsilon_{i}\right)$ is the greedy $q$-expansion of $\frac{1}{q-1}-\sigma$. Taking $x=\frac{1}{q-1}-\sigma, y=\sigma$ in Theorem 2.1, we get $\left(1-\varepsilon_{n+i}\right) \prec\left(\varepsilon_{i}\right)$ whenever $1-\varepsilon_{n}=0$, which shows that $\left(\varepsilon_{i}\right)$ is Usequence.

Remark. Theorem 4.1 is Lemma 2(b) in [2], but the proof here is different.

Theorem 4.2. If the greedy $q$-expansion $\left(\varepsilon_{i}\right)$ of $\sigma \in[0,1]$ with $q \in(1,2]$ is an $U$-sequence, then $\sigma$ has a unique $q$-expansion for this given $q$.

Proof. Assume the $q$-expansion $\left(\varepsilon_{i}\right)$ is a U-sequence. Then $\left(1-\varepsilon_{n+i}\right) \prec\left(\varepsilon_{i}\right)$ whenever $1-\varepsilon_{n}=0$. Since $\left(\varepsilon_{i}\right)$ is a $q$-expansion of $\sigma$, by the first part of the proof of Property L1, $\left(1-\varepsilon_{i}\right)$ is a $q$-expansion of $\frac{1}{q-1}-\sigma$. Being a U-sequence, $\left(\varepsilon_{i}\right)$ is infinite. Taking $y=\sigma \in[0,1], x=\frac{1}{q-1}-\sigma$ in Theorem 2.3(a), we deduce that $\left(1-\varepsilon_{i}\right)$ is the greedy $q$-expansion of $\frac{1}{q-1}-\sigma$. By Property L1, $\left(\varepsilon_{i}\right)$ is the lazy $q$-expansion of $\sigma$. Since $\left(\varepsilon_{i}\right)$ is both greedy and lazy, the number $\sigma$ has a unique $q$-expansion.

Remark. Taking $\sigma=1$ in Theorems 4.1 and 4.2, we get Theorem 2.2 in [5], which shows how special the number 1 is.

For certain real number $y \leq 1$, among base numbers $q$ for which $y$ has unique $q$-expansions, it is possible to determine the smallest such base $q$, which we now show.

Theorem 4.3. Let $\left(\delta_{i}\right) \subseteq\{0,1\}$ be defined recursively as follows:

- First set $\delta_{1}=1$.

- If $n \geq 0$ and if $\delta_{1}, \ldots, \delta_{2^{n}}$ are already defined, set $\delta_{2^{n}+k}=1-\delta_{k}$ for $1 \leq k<2^{n}$ and $\delta_{2^{n+1}}=1$. 
If $y \in\left[\sum_{i=1}^{\infty} \delta_{i} / 2^{i}, 1\right]$, then there is a smallest base $q \in(1,2]$ for which $y$ has a unique $U$-sequence $q$-expansion. This $q$ is the unique positive solution of the equation

$$
y=\sum_{i=1}^{\infty} \frac{\delta_{i}}{q^{i}}
$$

Proof. From Theorem 3 in [3], $\left(\delta_{i}\right)$ is the smallest U-sequence. For fixed $y \in$ $\left[\sum_{i=1}^{\infty} \delta_{i} / 2^{i}, 1\right]$, by Theorem 1.1, using $\left(\delta_{i}\right)=\left(e_{i}\right)$, there exists a unique $q \in$ $(1,2]$ for which $y=\sum_{i=1}^{\infty} \delta_{i} / q^{i}$. Using Theorem 2.3 (a) with $x=y,\left(e_{i}\right)=\left(a_{i}\right)=$ $\left(\delta_{i}\right)$, it follows that $\left(\delta_{i}\right)$ is the greedy $q$-expansion and so by Theorem $4.2, y$ has a unique $q$-expansion.

If $y$ has another U-sequence $q^{\prime}$-expansion $\left(e_{i}\right)$, which is also unique by the previous arguments, since $\left(\delta_{i}\right)$ is the smallest U-sequence, then $\left(e_{i}\right) \succeq\left(\delta_{i}\right)$ and Theorem 2.4 implies $q^{\prime} \geq q$.

\section{Numbers with exactly two $q$-expansions and smallest sequence}

We now proceed to find conditions for which there are exactly two $q$-expansions, which must then be greedy and lazy, of a positive number $y \leq 1$. Let $\left(e_{i}\right)$ be an infinite T-sequence. Since $\left(e_{i}\right)$ is also a D-sequence, then $e_{1}=1$; for otherwise applying (1.1) we would get $\left(e_{i}\right) \equiv(0)$, contradicting (1.1). From Theorem 1.1, for $y \in\left[\sum_{i=1}^{\infty} e_{i} / 2^{i}, \sum_{i=1}^{\infty} e_{i}\right)$, there exists a unique $q \in(1,2]$ satisfying

$$
\sum_{i=1}^{\infty} \frac{e_{i}}{q^{i}}=y
$$

By Theorem $2.3(\mathrm{a}),\left(e_{i}\right)$ is the greedy $q$-expansion of $y$. Let $m,\left(\varepsilon_{i}\right),\left(\delta_{i}\right)$ be as defined in the definition of T-sequence. Assume further that $\left(\varepsilon_{i}\right)$ is a $q$-expansion of 1 . Thus

$$
\sum_{i=1}^{\infty} \frac{\delta_{i}}{q^{i}}=\sum_{i<m} \frac{e_{i}}{q^{i}}+\sum_{i>m} \frac{e_{i}+\varepsilon_{i-m}}{q^{i}}=\sum_{i \neq m} \frac{e_{i}}{q^{i}}+\frac{1}{q^{m}} \sum_{i=1}^{\infty} \frac{\varepsilon_{i}}{q^{i}}=\sum_{i=1}^{\infty} \frac{e_{i}}{q^{i}}=y,
$$

showing that $\left(\delta_{i}\right)$ is also a $q$-expansion of $y$. Notice that the $q$-expansions $\left(e_{i}\right)$ and $\left(\delta_{i}\right)$ are different because $e_{m}=1$ but $\delta_{m}=0$.

Theorem 5.1. Let $\left(e_{i}\right)$ be an infinite T-sequence with corresponding $m,\left(\varepsilon_{i}\right)$, $\left(\delta_{i}\right)$. For $y \in\left[\sum_{i=1}^{\infty} e_{i} / 2^{i}, 1\right]$, let $q \in(1,2]$ be the unique base, as guaranteed by Theorem 1.1, such that $y=\sum_{i=1}^{\infty} e_{i} / q^{i}$. Assume $\left(\varepsilon_{i}\right)$ is a q-expansion of 1 . Then $y$ has exactly two different q-expansions, given by (5.1) and (5.2).

Proof. From what mentioned above, $\left(e_{i}\right)$ is the greedy $q$-expansion of $y$. On the other hand, from (1.3) and Theorem 3.2, we see that $\left(\delta_{i}\right)$ is the lazy $q$-expansion of $y \leq 1$. It remains to verify that if a sequence $\left(\rho_{i}\right) \subseteq\{0,1\}$ satisfies the strict 
inequalities $\left(\delta_{i}\right) \prec\left(\rho_{i}\right) \prec\left(e_{i}\right)$, then

$$
\sum_{i=1}^{\infty} \frac{\rho_{i}}{q^{i}} \neq y
$$

Fix such a sequence $\left(\rho_{i}\right)$. Then $\rho_{i}=\delta_{i}=e_{i}$ for all $i<m$. Since $\delta_{m}=0$ and $e_{m}=1$, we have either $\rho_{m}=\delta_{m}=0$ or $\rho_{m}=e_{m}=1$.

Case 1: $\rho_{m}=0$. Then there is an integer $n>m$ such that $\rho_{i}=\delta_{i}$ for all $i<n$ and $\delta_{n}=0<1=\rho_{n}$. Using the properties of T-sequence and the same arguments as in the proof of Theorem 2.3 (a) up to equation (2.3), we deduce $\sum_{i=1}^{\infty} \delta_{n+i} / q^{i}<1$. Thus

$$
\sum_{i=1}^{\infty} \frac{\rho_{i}}{q^{i}}-y=\frac{1}{q^{n}}+\sum_{i>n} \frac{\rho_{i}-\delta_{i}}{q^{i}} \geq \frac{1}{q^{n}}-\sum_{i>n} \frac{\delta_{i}}{q^{i}}=\frac{1}{q^{n}}\left(1-\sum_{i=1}^{\infty} \frac{\delta_{n+i}}{q^{i}}\right)>0,
$$

proving (5.3).

Case 2: $\rho_{m}=1$. Then there is an integer $n>m$ such that $\rho_{i}=e_{i}$ for all $i<n$ and $\rho_{n}=0<1=e_{n}$. Using the properties of T-sequence and the same arguments as in the first case, we have $\sum_{i=1}^{\infty} \overline{e_{n+i}} / q^{i}<1$. Hence

$\sum_{i=1}^{\infty} \frac{\rho_{i}}{q^{i}}-y=-\frac{1}{q^{n}}+\sum_{i>n} \frac{\rho_{i}-e_{i}}{q^{i}} \leq-\frac{1}{q^{n}}+\sum_{i>n} \frac{\overline{e_{i}}}{q^{i}}=-\frac{1}{q^{n}}\left(1-\sum_{i=1}^{\infty} \frac{\overline{e_{n+i}}}{q^{i}}\right)<0$, again implying (5.3).

Remark. Theorem 3.1 in [5] is a special case of Theorem 5.1 above when $y=1$.

Theorem 5.2. Let $\left(e_{i}\right)$ be a finite T-sequence with $e_{L}$ being its last nonzero digit and corresponding $m,\left(\varepsilon_{i}\right),\left(\delta_{i}\right)$. For $y \in\left[\sum_{i=1}^{\infty} e_{i} / 2^{i}, 1\right]$, let $q \in(1,2]$ be the unique base such that $y=\sum_{i=1}^{\infty} e_{i} / q^{i}$. Assume that $\left(\varepsilon_{i}\right)$ is the greedy q-expansion of 1 and that

$$
\begin{aligned}
& L>\min \left\{k ; \text { for each } i \in \mathbb{N} \text {, if } \delta_{i}=1, \text { then } \delta_{i+j} \neq e_{j} \text { when } 1 \leq j<k\right. \\
& \text { and } \left.\delta_{i+k}=e_{k}=1\right\} .
\end{aligned}
$$

Then $y$ has exactly two different q-expansions, given by (5.1) and (5.2).

Proof. The proof proceeds exactly as in Theorem 5.1, except that now, at the beginning of the proof, we appeal to Theorem 3.3 instead of Theorem 3.2.

Remark. Theorem 5.2 is new and complements Theorem 5.1.

As an example for Theorem 5.2, take

$$
y=\frac{3902563888221395449817251061561905663982412670490}{3914144333903073791808962606796280957916632792441}
$$

and $q=1.9$. Here

$\left(e_{i}\right)=(1,1,1,0,1,0,0,1,0,1,0,0,0,0,0,0,1,0,0,0,0,0,0,0,0,0,0,0,0,0,0,0,0,0,0,0,0,1)$ 
is a $q$-expansion of $y$,

$\left(\varepsilon_{i}\right)=(1,1,1,0,1,0,0,1,1,0,1,1,0,1,1,0,0,0,1,0,1,1,1,0,0,1,0,0,1,1,0,0,1,0,0,1,1,0,0,0,1,0,1,1,0,0,1,1,0,1,0,1, \ldots)$

is the greedy $q$-expansion of $1, m=10$ and $e_{i+m}+\varepsilon_{i} \in\{0,1\}(i \geq 1)$.

Let $\left(e_{i}\right)$ be an infinite T-sequence with corresponding $m,\left(\varepsilon_{i}\right)$ and $\left(\delta_{i}\right)$. For a given real number $y$ in an appropriate range, under the hypotheses of Theorem 5.1, $y$ has exactly two $q$-expansions, namely the greedy $\left(e_{i}\right)$ and the lazy $\left(\delta_{i}\right)$. The corresponding base $q$ is then a T-base number. We now ask the question: given the real number $y$ in an appropriate range what is its smallest, with respect to lexicographic order, T-sequence? An answer is given in the next theorem.

Theorem 5.3. Let $\left(e_{i}^{\prime}\right)=111 \underline{001}$, the symbol 001 denoting the period 001 of a periodic sequence. If $\left(e_{i}\right)$ is an infinite T-sequence $q$-expansion of $y \in$ $\left[\sum_{i=1}^{\infty} e_{i} / 2^{i}, 1\right] \cap\left[\sum_{i=1}^{\infty} e_{i}^{\prime} / 2^{i}, 1\right]$ which begins with 111 with corresponding $m>$ 3 not a multiple of 3 , and $\left(\varepsilon_{i}\right)$ being the greedy $q$-expansion of 1 , then $q \geq q^{\prime}$, where $q^{\prime} \in(1,2]$ is the unique real number satisfying $\sum_{i=1}^{\infty} e_{i}^{\prime} /\left(q^{\prime}\right)^{i}=y$.

Proof. By Theorem 2.4, it suffices to show that $\left(e_{i}\right) \succeq\left(e_{i}^{\prime}\right)$. Assume

$$
\left(e_{i}\right) \prec\left(e_{i}^{\prime}\right)=111 \underline{\underline{001}} \text {. }
$$

Thus $\left(e_{i}\right)$ takes the form

$$
\underbrace{111}_{1} \underbrace{001}_{2} \cdots \underbrace{001}_{k} 000 \cdots \text { for some } k \geq 2
$$

or 111000 which may be treated as (5.5) with $k=1$. From (1.2) the sequence $\left(\delta_{i}\right)$ also begins with (5.5), i.e., $\delta_{i}: 111001 \cdots 001000$. Applying (1.3), we conclude that $\delta_{3 k+4}=\delta_{3 k+5}=1$ (because $\delta_{3 k}=1$ ). Therefore, the sequence $\left(e_{i}\right)$ also begins with $111001 \cdots 00100011$. We distinguish two cases.

Case 1: If $e_{3 k+6}=1$, then $\delta_{3 k+6}=1$ (since $m$ cannot is a multiple of 3 ). From (1.1), $e_{3 k+7}=e_{3 k+8}=0$ (because $e_{3 k+3}=0$ ).

Subcase 1.1: If $e_{3 k+9}=1$, then $\delta_{3 k+9}=1$ (since $m$ cannot is a multiple of 3). From (1.1), $e_{3 k+10}=e_{3 k+11}=0$ (because $e_{3 k+3}=0$ ). The step now repeats as in Case 1.

Subcase 1.2: If $e_{3 k+9}=0$, then $\delta_{3 k+9}=0$ (because of $3 k+9<m$ and (5.2)) The step then repeats as in Case 2 .

Case 2: If $e_{3 k+6}=0$, then $\delta_{3 k+6}=0$ (because of $3 k+6<m$ and (5.2)). From (1.3), $\delta_{3 k+7}=\delta_{3 k+8}=1$ (because $\delta_{3 k}=1$ ). Thus $e_{3 k+7}=e_{3 k+8}=1$ (because of $3 k+8<m$ and (5.2)). The step now repeats as in Case 1 .

Subcase 2.1: If $e_{3 k+9}=1$, then $\delta_{3 k+9}=1$ (since $m$ cannot is a multiple of $3)$.

Subcase 2.2: If $e_{3 k+9}=0$, then $\delta_{3 k+9}=0$ (because of $3 k+9<m$ and (5.2)). From $(1.3), \delta_{3 k+10}=\delta_{3 k+11}=1$ (because $\delta_{3 k}=1$ ). The step repeats as in Case 2.

Continuing in the same manner, we deduce that $m$ must be arbitrarily large, which is impossible. 
Remark. Theorem 4.1 in [5] is a special case of Theorem 5.3 when $y=1$.

As an example of Theorem 5.3, let

$$
y=\frac{3902563888221395449817251061561905663982412670490}{3914144333903073791808962606796280957916632792441}
$$

and $q=1.9$. The unique positive solution of the equation $\sum_{i=1}^{\infty} e_{i}^{\prime} /\left(q^{\prime}\right)^{i}=y$ is $q^{\prime} \approx 1.874535175$. From Theorem 4.1 in [5], when $y=1$ we have $q^{\prime}=$ 1.871349313 .

The last two results show that for certain $y \leq 1$, the sequence $\left(e_{i}^{\prime}\right)$ with base $q^{\prime}$ yields a unique $q^{\prime}$-expansion whose base is an accumulation point of, yet smaller than, other T-base numbers $q$ of $y$ with exactly two $q$-expansions.

Theorem 5.4. Let $\left(e_{i}^{\prime}\right)=111 \underline{\underline{001}}$. For $y \in\left[\sum_{i=1}^{\infty} e_{i}^{\prime} / 2^{i}, 1\right]$, there is a unique $q^{\prime} \in(1,2]$ such that $\left(e_{i}^{\prime}\right)$ is a $q^{\prime}$-expansion of $y$ and this $q^{\prime}$-expansion is always unique.

Proof. Taking both sequences to be $\left(e_{i}^{\prime}\right)$ in Theorem 2.3 (a), we have that $\left(e_{i}^{\prime}\right)$ is the greedy $q^{\prime}$-expansion of $y$. Since $\left(e_{i}^{\prime}\right)$ is also a U-sequence, Theorem 4.2 infers that $y$ has a unique $q^{\prime}$-expansion.

Theorem 5.5. Let $\left(e_{i}^{\prime}\right)=111 \underline{\underline{001}}, y \in\left[\sum_{i=1}^{\infty} e_{i}^{\prime} / 2^{i}, 1\right]$. By Theorem 1.1, there exists a unique $q^{\prime} \in(1,2]$ such that $y=\sum_{i=1}^{\infty} e_{i}^{\prime} /\left(q^{\prime}\right)^{i}$. Let $k \in \mathbb{N}$ and let

$$
\left(e_{i}^{(k)}\right):=111 \overbrace{001}^{1} \ldots \overbrace{001}^{k} 1 \underbrace{0 \cdots 0}_{3 k+4} 001001001001 \ldots
$$

be the sequence obtained by inserting the block $10 \cdots 0$ (one 1 followed by $(3 k+$ 4) 0's) between the $k^{\text {th }}$ and $(k+1)^{\text {th }}$ block of 001 of $\left(e_{i}^{\prime}\right)$. By Theorem 1.1, there exists a unique $q_{k} \in(1,2]$ such that $y=\sum_{i=1}^{\infty} e_{i}^{(k)} / q_{k}^{i}$. Let $\varepsilon_{i}^{(k)}$ be the greedy $q_{k}$-expansion of 1 . Assume there are infinitely many $k$ such that

$$
\begin{aligned}
& \left(\overline{\varepsilon_{n+i}^{(k)}}\right) \prec\left(e_{i}^{(k)}\right) \text { whenever } \varepsilon_{n}^{(k)}=1 \text { and } 1 \leq n \leq 3 k+3, \\
& \left(\varepsilon_{n+i}^{(k)}\right) \prec\left(e_{i}^{(k)}\right) \text { whenever } \varepsilon_{n}^{(k)}=0 \text { and } 1 \leq n \leq 3 k+4, \\
& \varepsilon_{3 k+4+3 \mathbb{N}}^{(k)}=0 \text { where } 3 \mathbb{N}=\{3 t ; t \in \mathbb{N}\}, \\
& \varepsilon_{3 k+2+3 \mathbb{N}}^{(k)} \varepsilon_{3 k+3+3 \mathbb{N}}^{(k)} \neq 11, \\
& \varepsilon_{3 k+2+3 \mathbb{N}}^{(k)} \varepsilon_{3 k+3+3 \mathbb{N}}^{(k)}, \varepsilon_{3 k+5+3 \mathbb{N}}^{(k)} \varepsilon_{3 k+6+3 \mathbb{N}}^{(k)} \neq 01,10 .
\end{aligned}
$$

Then $q^{\prime}$ is an accumulation point of the set of T-base numbers.

Proof. We start by verifying that $\left(e_{i}^{(k)}\right)$ is a T-sequence with $m=3 k+4$ and $\left(\delta_{i}^{(k)}\right) \subseteq\{0,1\}$ so constructed as in (1.2) with corresponding $\left(e_{i}^{(k)}\right)$ and $\left(\varepsilon_{i}^{(k)}\right)$; such construction is valid by (5.8). 
From the shape of the sequence $\left(e_{i}^{(k)}\right)$, we see that $\left(e_{i}^{(k)}\right)$ is a D-sequence and $e_{3 k+4}^{(k)}=1$. There remains to check the requirements (1.3), (1.4) and (1.5). The requirement (1.4) follows immediately from the shape of the sequence $\left(e_{i}^{(k)}\right)$.

When $i<m=3 k+4$, since $\delta_{i}^{(k)}=e_{i}^{(k)}$, the requirement (1.3) holds for these $i$. From (5.6), respectively (5.7), together with the definition $(1.2),\left(\delta_{i}^{(k)}\right)$ satisfies (1.3), respectively (1.5), when $m+1 \leq n \leq m+3 k+4$.

For $n \geq m+3 k+5,(1.3)$ holds by the definition (1.2) and the shape of $\left(e_{i}^{(k)}\right)$. As for (1.5), we distinguish four cases.

Case 1: $\delta_{m+3 k+2+3 \mathbb{N}}^{(k)} \delta_{m+3 k+3+3 \mathbb{N}}^{(k)}=00$. From (5.9),

$$
\delta_{m+3 k+5+3 \mathbb{N}}^{(k)} \delta_{m+3 k+6+3 \mathbb{N}}^{(k)} \neq 11
$$

i.e., $\left(\delta_{i}^{(k)}\right)$ satisfies $(1.5)$.

Case 2: $\delta_{m+3 k+2+3 \mathbb{N}}^{(k)} \delta_{m+3 k+3+3 \mathbb{N}}^{(k)}=01$. From (5.9),

$$
\delta_{m+3 k+5+3 \mathbb{N}}^{(k)} \delta_{m+3 k+6+3 \mathbb{N}}^{(k)} \neq 11,
$$

while from $(5.10), \delta_{m+3 k+5+3 \mathbb{N}}^{(k)} \delta_{m+3 k+6+3 \mathbb{N}}^{(k)} \neq 10$, i.e., $\left(\delta_{i}^{(k)}\right)$ satisfies $(1.5)$.

Case 3: $\delta_{m+3 k+2+3 \mathbb{N}}^{(k)} \delta_{m+3 k+3+3 \mathbb{N}}^{(k)}=10$. From (5.9),

$$
\delta_{m+3 k+5+3 \mathbb{N}}^{(k)} \delta_{m+3 k+6+3 \mathbb{N}}^{(k)} \neq 11,
$$

i.e., $\left(\delta_{i}^{(k)}\right)$ satisfies $(1.5)$.

Case $4: \delta_{m+3 k+2+3 \mathbb{N}}^{(k)} \delta_{m+3 k+3+3 \mathbb{N}}^{(k)}=11$. That $\left(\delta_{i}^{(k)}\right)$ satisfies (1.5) follows at once from (5.9).

Since $\left(e_{i}^{(k)}\right)$ is a T-sequence, taking $k \rightarrow \infty$, we have $\left(e_{i}^{(k)}\right) \rightarrow\left(e_{i}^{\prime}\right)$ and the corresponding base numbers $q_{k} \rightarrow q^{\prime}$, which completes the proof.

\section{References}

[1] K. Dajani and M. de Vries, Measures of maximal entropy for random $\beta$-expansions, J. Eur. Math. Soc. (JEMS) 7 (2005), no. 1, 51-68.

[2] K. Dajani and C. Kraaikamp, From greedy to lazy expansions and their driving dynamics, Expo. Math. 20 (2002), no. 4, 315-327.

[3] P. Erdös, M. Horváth, and I. Joó, On the uniqueness of the expansions $1=\sum q^{-n_{i}}$, Acta Math. Hungar. 58 (1991), no. 3-4, 333-342.

[4] P. Erdös, I. Joó, and V. Komornik, Characterization of the unique expansions $1=$ $\sum_{i=1}^{\infty} q^{-n_{i}}$ and related problems, Bull. Soc. Math. France 118 (1990), no. 3, 377-390.

[5] V. Komornik and P. Loreti, Unique developments in non-integer bases, Amer. Math. Monthly 105 (1998), no. 7, 636-639.

[6] _ On the expansions in non-integer bases, Rend. Mat. Appl. (7) 19 (1999), no. 4, 615-634.

[7] N. Sidorov, Expansions in non-integer bases: lower, middle and top orders, J. Number Theory 129 (2009), no. 4, 741-754. 
Danita Chunarom

Department of Mathematics

Kasetsart University

BANGKOK 10900, THAILAND

E-mail address: dchun@ipst.ac.th

VICHIAN LAOHAKOSOL

Department of Mathematics

Kasetsart University

BANGKOK 10900, THAILAND

E-mail address: fscivil@ku.ac.th 